\title{
THE FIRST INITIAL-BOUNDARY VALUE PROBLEM FOR SOME NONLINEAR TIME DEGENERATE PARABOLIC EQUATIONS
}

\author{
MARGARET C. WAID ${ }^{1}$
}

Abstract. Consider the nonuniformly parabolic operator

$$
L u=\sum_{i, j=1}^{n} a^{i j}(x, t) u_{x_{i} x_{j}}+\sum_{i=1}^{n} b^{i}(x, t) u_{x_{i}}-c(x, t, u) u_{t}+d(x, t) u,
$$

where $u, a^{i j}, b^{i}, c, d$ are bounded, real-valued functions defined on a domain $D=\Omega \times[0, T] \subset R^{n+1}$. Assume that $c(x, t, u)$ is Lipschitz continuous in $\left.\left.\right|^{-}\right|_{\alpha} ^{D}$ of $C_{\alpha}(D)$, and that $c(x, t, u) \geqq 0$ on $D$. Sufficient conditions on $c$ are found which guarantee existence of a unique solution $u \in \bar{C}_{2+\alpha}$ to the first initial-boundary value problem $L u=f(x, t), u=\psi$, on the normal boundary of $D$, where $\psi \in \bar{C}_{2+\alpha}$. Existence is proved by direct application of a fixed point theorem due to Schauder using existence of a solution to the linear problem as well as a priori estimates.

1. Introduction. We shall be concerned with existence of a solution to the first initial-boundary value problem for the second-order nonlinear nonuniformly parabolic operator

$$
L u=\sum_{i, j=1}^{n} a^{i j} u_{x_{i} x_{j}}+\sum_{i=1}^{n} b^{i}(x, t) u_{x_{i}}-c(x, t, u) u_{t}+d(x, t) u,
$$

where $u$ and all coefficients of $L$ are real-valued functions defined for $(x, t)=\left(x_{1}, \cdots, x_{n}, t\right)$ in an $(n+1)$-dimensional, bounded, convex domain $D$. Subscripts will be used to denote differentiation.

We will assume that $L$ is parabolic; that is,

$$
\sum_{i, j=1}^{n} a^{i j} \xi_{i} \xi_{j} \geqq \gamma|\xi|^{2}>0
$$

\footnotetext{
Received by the editors April 9, 1973.

AMS (MOS) subject classifications (1970). Primary 35K15, 35K20, 35K60; Secondary 35M05, 76S05.

Key words and phrases. Degenerate parabolic equation, nonlinear parabolic operators, nonuniformly parabolic operators, existence theorems for nonlinear equations, applications of fixed point theorems.

${ }^{1}$ This research was supported in part by the University of Delaware Research Foundation.
} 
for some $\gamma>0$ and for any real vector $\xi \neq 0$. Assume that the coefficient $c(x, t, u) \geqq 0$ but is not necessarily bounded away from zero. Since $c(x, t, u)$ may be zero for some $(x, t) \in D, L$ may be a degenerate parabolic operator. Note that $c$ may be a function of $u$ as well as of $x$ and $t$.

The author has already proved the existence of a unique solution to the first initial-boundary value problem for the linear equation

$$
M u=\sum_{i, j=1}^{n} a^{i j} u_{x_{i} x_{j}}+\sum_{i=1}^{n} b^{i} u_{x_{i}}-c(x, t) u_{t}+d(x, t) u=f(x, t)
$$

in [4]. Existence of a solution to the nonlinear problem is proved with the aid of a fixed point theorem due to Schauder. We shall also use the fact that we can obtain a solution to the linear initial-boundary value problem together with a priori estimates obtained in [4].

This partial differential equation arises in applications to studies dealing with fluid flow through porous media. One such application [3] deals with diffusion and filtration of lipid-protein complexes and oxygen through the arterial wall and into surrounding tissue. Historically, the porosity coefficient $c$ has been assumed to be a positive constant, which is an average obtained in studying a healthy artery. However, tissue, as well as blood, is a heterogeneous composite of materials. In the case of a nonhealthy artery (as in atherosclerosis), it is precisely this heterogeneity which is of interest. The coefficient of porosity $c(x, t, u)$ is more accurately and usefully described as a coefficient of space, time, and pressure.

2. Notation and the linear first initial-boundary value problem. We shall use the same notation as A. Friedman [2, p. 40] to describe the domain $D$. As stated in the introduction, $D$ is a bounded, convex, $(n+1)$ dimensional domain in $R^{n+1}$, where $(x, t)=\left(x_{1}, \cdots, x_{n}, t\right)$ represents a variable point in $R^{n+1}$. $\partial D$ denotes the boundary of $D$. Thus, $\partial D=\bar{B}+$ $B_{T}+S$, where $B$ is a domain in $R^{n} \times\{0\}, B_{T}(T>0)$ is a domain in $R^{n} \times\{T\}$, and $S$ is a manifold, not necessarily connected, in $R^{n} \times(0, T] . S+\bar{B}$ therefore denotes the parabolic, or normal, boundary of $D$.

Let $D_{\tau}=D \cap\left(R^{n} \times(0, \tau)\right), B_{\tau}=D \cap\left(R^{n} \times\{\tau\}\right)$, and $S_{\tau}=S \cap\left(R^{n} \times(0, \tau]\right)$. Assume that for each $\tau \in(0, T), B_{\tau}$ is a domain. Then, for every $(x, \tau)$ in $D, 0<\tau<T$, if $S(x, \tau)=D_{\tau}+B_{\tau}$, then $(S(x, \tau))-S(x, \tau)=\bar{B}+S_{r}$, where $(S(x, \tau))$ denotes the closure of $(S(x, \tau))$. Also, assume that the following is a condition on $D$ : There exists a simple continuous curve $\alpha$ connecting $B$ to $B_{T}$ along which the $t$-coordinate is nondecreasing.

Introduce the metric $d$ defined by

$$
d(P, Q)=\left[|x-\bar{x}|^{2}+|t-\bar{t}|\right]^{1 / 2}
$$


where $P=(x, t), Q=(\bar{x}, \bar{t})$, and $|x|=\left(\sum_{i=1}^{n} x_{i}^{2}\right)^{1 / 2}$. Hölder continuity of a function $f$ is defined with respect to the metric $d$.

Suppose $\alpha \in(0,1)$. Then let

$|u|_{0}^{D}=\sup _{D}|u|, \quad \bar{H}_{\alpha}^{D}(u)=\sup _{P, Q \in D} \frac{|u(P)-u(Q)|}{d(P, Q)^{\alpha}}, \quad|\bar{u}|_{\alpha}^{D}=|u|_{0}^{D}+\bar{H}_{\alpha}^{D}(u)$.

Then, $\bar{C}_{\alpha}(D)=\left\{u: u: \bar{D} \rightarrow R,|\bar{u}|_{\alpha}^{D}<\infty\right\}$ is a Banach space with norm $\left.1^{\circ}\right|_{\alpha} ^{D}$; see [2, pp. 62-63].

Denote by $D_{x}^{m}$ any partial derivative of order $m$ with respect to the variables $x_{1}, \cdots, x_{n}$ and let $D_{t}=\partial / \partial t$. If $D_{x} u, D_{x}^{2} u, D_{t} u$ exist in $D$, then we define

$$
|\bar{u}|_{2+\alpha}^{D}=|\bar{u}|_{\alpha}^{D}+\sum\left|\left(D_{x} u\right)-\right|_{\alpha}^{D}+\sum\left|\left(D_{x}^{2} u\right)-\right|_{\alpha}^{D}+\left|\left(D_{t} u\right)-\right|_{\alpha}^{D},
$$

where the sums are taken over all partial derivatives of the indicated order. Let

$$
\bar{C}_{2+\alpha}(D)=\left\{u: u: \bar{D} \rightarrow R,|\bar{u}|_{2+\alpha}^{D}<\infty\right\} .
$$

Then, $\bar{C}_{2+\alpha}(D)$ is a Banach space with norm $|\cdot|_{2+\alpha}^{D}$; see [2]. When there is no confusion, we will drop the $D$ from $\left.\left.\right|^{*}\right|_{\alpha} ^{D},\left.\left.\right|^{-}\right|_{2+\alpha} ^{D}$.

Definition 2.1. We say that $D$ has property $(\bar{E})$ if for every point $Q$ of $\bar{S}$, there exists an $(n+1)$-dimensional neighborhood $V$ such that $V \cap \bar{S}$ can be represented, for some $i(1 \leqq i \leqq n)$, in the form

$$
x_{i}=h\left(x_{1}, \cdots, x_{i-1}, x_{i+1}, \cdots, x_{n}, t\right),
$$

where $h, D_{x} h, D_{x}^{2} h, D_{t} h$ are Hölder continuous of exponent $\alpha$.

Definition 2.2. If $D$ has property $(\bar{E})$ and if the functions $D_{x} D_{t} h$, $D_{t}^{2} h$ of the local representations of $S$ exist and are continuous functions, then we say $D$ has property $\left(E^{\prime}\right)$.

Definition 2.3. A function $\psi$ defined on $\bar{B}+S$ is said to belong to $\bar{C}_{2+\alpha}(D)$ if there exist functions $\Psi$ in $\bar{C}_{2+\alpha}(D)$ such that $\Psi=\psi$ on $\bar{B}+S$. Then $|\bar{\psi}|_{2+\alpha}^{D}$ is defined by

$$
|\bar{\psi}|_{2+\alpha}^{D}=\inf _{\Psi}|\bar{\Psi}|_{2+\alpha}^{D}
$$

where the infimum is taken over all $\Psi \in \bar{C}_{2+\alpha}(D)$ which coincide with $\psi$ on $\bar{B}+S$.

The following notation is one of convenience. If $u$ is a bounded realvalued function on a subset $S$ of $R^{n+1}$, define

and

$$
M(u ; S)=\sup \{u(x, t):(x, t) \in S\}
$$

$$
m(u ; S)=\inf \{u(x, t):(x, t) \in S\} .
$$


In [4], the author solves the linear first initial-boundary value problem

$$
\begin{aligned}
N u & =\sum_{i, j=1}^{n} a^{i j} u_{x_{i} x_{j}}+\sum_{i=1}^{n} b^{i}(x, t) u_{x_{i}}-c(x, t) u_{t}+d(x, t) u \\
& =f(x, t) \text { on } D+B_{T} \\
u & =\psi \text { on } \bar{B}+S
\end{aligned}
$$

without assuming that $c$ has a positive minimum in $D$. We now state this theorem precisely.

THEOREM 2.4. Assume that $a^{i j}$ is constant for each $i, j$, that all coefficients of $N$, defined in (2.1) are of class $C^{1,1}(\bar{D}), u \in C(\bar{D}), u \in C^{3}(D)$, and $a^{11} \lambda^{2}+b^{1} \lambda \geqq 1$ for some $\lambda>0$. Suppose, further, that the coefficients of $N$ are uniformly Hölder continuous (exponent $\alpha)$ in $D,\left|\left(a^{i j}\right)-\right|_{\alpha} \leqq K_{1}$, $\left|\left(b^{i}\right)-\right|_{\alpha} \leqq R_{1},|\bar{c}|_{\alpha} \leqq R_{1},|\vec{d}|_{\alpha} \leqq K_{1}$, that $m(c ; \bar{B}+S) \geqq \mu>0$ while $m(c ; D) \geqq 0$, that $|\bar{f}|_{\alpha}<\infty$, and that (1.2) holds. If $D$ has property $\left(E^{\prime}\right), \psi \in \bar{C}_{2+\alpha}(D)$, and $N \psi=f$ on $\partial B$, then there exists a unique solution $u$ of the first initialboundary value problem (2.1) and, furthermore, $u \in \bar{C}_{2+\alpha}(D)$.

3. A priori estimates. Our proof for the nonlinear case requires the use of an a priori estimate for a solution to the linear problem. The following theorem was proved in [4].

THEOREM 3.1. Suppose that the conditions of Theorem 2.4 hold, together with the added restriction that $m(c ; D) \geqq \mu>0$. Then there exists a constant $\bar{K}$ depending only on $\bar{K}_{1}, K_{2}, \alpha$, and $D$ such that if $u$ is a solution to $N u=f$, with $u=\psi$ on $\bar{B}+S$, and if $u \in \bar{C}_{2+\alpha}(D)$, then

$$
|\bar{u}|_{2+\alpha} \leqq \mu^{-1 / 2} \bar{K}\left(|\bar{\psi}|_{2+\alpha}+|\bar{f}|_{\alpha}\right) .
$$

The technique which was used in obtaining the solution in Theorem 2.4 involved perturbing the coefficient $c$ by $1 / k$ and considering the problem

$$
\begin{aligned}
N^{k} u & =N u-(1 / k) u_{t}=f \text { on } D+B_{T}, \\
u & =\psi \quad \text { on } \bar{B}+S .
\end{aligned}
$$

By Friedman's work, we were guaranteed a unique solution $u_{k} \in \bar{C}_{2+\alpha}(D)$. We were able to show that the sequence $\left\{u_{k}\right\}$ obtained in this manner is Cauchy in the Banach space $\bar{C}_{2+\alpha}(D)$ and does, in fact, converge to the unique solution of the first initial-boundary value problem (2.1).

These $u_{k}$, incidentally, satisfy

$$
\left|\left(u_{k}\right)-\right|_{2+\alpha} \leqq k^{1 / 2} R\left(|\bar{\psi}|_{2+\alpha}+|\bar{f}|_{\alpha}\right)
$$


If $|\bar{\psi}|_{2+\alpha}+|f|_{\alpha}>0$, there is some $u_{K}$ such that $\left|\left(u-u_{K}\right)^{-}\right|_{2+\alpha}<|\bar{\psi}|_{2+\alpha}+$ $|\bar{f}|_{\alpha}$. Since $u_{K}$ satisfies (3.1), we may conclude that

$$
\begin{aligned}
|\bar{u}|_{2+\alpha} & =\left|\left(u-u_{K}+u_{K}\right)-\right|_{2+\alpha} \leqq 1\left(u-u_{K}\right)-\left.\right|_{2+\alpha}+\left|\left(u_{K}\right)-\right|_{2+\alpha} \\
& \leqq\left(|\bar{\psi}|_{2+\alpha}+|\bar{f}|_{\alpha}\right)+K^{1 / 2} \bar{K}\left(|\bar{\psi}|_{2+\alpha}+|\bar{f}|_{\alpha}\right) \\
& =\left(1+K^{1 / 2} R\right)\left(|\bar{\psi}|_{2+\alpha}+|\bar{f}|_{\alpha}\right) .
\end{aligned}
$$

We have just proved the following theorem.

THEOREM 3.2. Assume all the conditions of Theorem 2.4. Then the solution $u$ of the first initial-boundary value problem (2.1) satisfies

$$
|\bar{u}|_{2+\alpha} \leqq \bar{M}\left(|\bar{\psi}|_{2+\alpha}+|\bar{f}|_{\alpha}\right) \text {, }
$$

for some constant $\bar{M}$ which is independent of $m(c ; D)$, provided $|\bar{\psi}|_{2+\alpha}+|\bar{f}|_{\alpha}>$ 0 .

4. The nonlinear problem. We now proceed to solve the first initialboundary value problem ( $L$ defined as in (1.1)):

$$
\begin{aligned}
L u & =f(x, t), \quad \text { on } D+B_{T}, \\
u & =\psi, \quad \text { on } \bar{B}+S .
\end{aligned}
$$

For $v$ a fixed element of $\bar{C}_{2+\alpha}(D)$, consider the linear problem

$$
\begin{aligned}
L_{v} u & =\sum_{i, j=1}^{n} a^{i j} u_{x_{i} x_{j}}+\sum_{i=1}^{n} b^{i} u_{x_{i}}-c(x, t, v) u_{t}+d u \\
& =f(x, t), \quad \text { on } D+B_{T} \\
u & =\psi, \text { on } \bar{B}+S .
\end{aligned}
$$

Assuming that as a function of $x$ and $t, c(x, t, v)$ satisfies the hypotheses of Theorem 2.4, we obtain a solution $u \in \bar{C}_{2+\alpha}(D)$ to the initial-boundary value problem (4.2). $u$ obviously depends upon $v$. Hence, we define $\phi: \bar{C}_{2+\alpha}(D) \rightarrow \bar{C}_{2+\alpha}(D)$ by $u=\phi(v)$ is the unique solution to the initialboundary value problem (4.2).

Under appropriate conditions on $c$, we will show that

(i) $\phi: A \rightarrow A$, where $A$ is some closed convex subset of $\bar{C}_{2+\alpha}(D)$;

(ii) $\phi$ is continuous in $\left.\left.\right|^{-}\right|_{2+\alpha} ^{D}$ on $A$.

We will then be able to apply the Schauder fixed point theorem of [2, p. 189] to obtain an element $u \in \bar{C}_{2+x}(D)$ such that $\phi(u)=u$. Then $u$ will be a solution to (4.1) and we will have proved the desired existence theorem.

An argument similar to that given by Friedman in [2] allows us to assume that $\psi \equiv 0$ on $\bar{B}+S$. We will assume that $c(x, t, u)$ is Lipschitz continuous with respect to $u$ in the $\left.\left.\right|^{\top}\right|_{\alpha}$ norm. That is, if $(x, t) \in D$ 
and $u_{1}, u_{2} \in C_{\alpha}(D)$, then

$$
\left|\left(c\left(x, t, u_{1}\right)-c\left(x, t, u_{2}\right)\right)-\right|_{a}^{D} \leqq \eta\left|\left(u_{1}-u_{2}\right)-\right|_{\alpha}^{D}
$$

for some positive constant $\eta$.

Let $A=\left\{u:|\bar{u}|_{2+\alpha}^{D} \leqq 2 \bar{M}|\bar{f}|_{\alpha}^{D}, u=0\right.$ on $\left.\bar{B}+S\right\}$ where $f$ is the forcing function in (4.2) and $\bar{M}$ is the constant obtained in Theorem 3.2. $A$ is clearly a closed convex subset of $\bar{C}_{2+\alpha}(D)$.

Now suppose that $v \in A$ and $h \in[0,1]$. Then $\phi(h v)$ satisfies $L_{h v} \phi(h v)=f$, $\phi(h v)=0$ on $\bar{B}+S$. We note that $L_{h v} \phi(h v)=f$ can be rewritten in the form

$$
L_{v} \phi(v)=L_{v} \phi(v)-L_{h v} \phi(h v)+f \equiv F(v) .
$$

Hence, $\phi(v)$ satisfies:

$$
\begin{array}{clrl}
L_{v} \phi(v) & =F(v) & & \text { in } D+B_{T}, \\
\phi(v) & =0 & & \text { on } \bar{B}+S .
\end{array}
$$

Using (4.3), we see that

$$
\begin{aligned}
|(F(v))-|_{\alpha}^{D} \leqq & \left|\left(L_{v} \phi(v)-L_{h v} \phi(h v)\right)-\right|_{\alpha}^{D}+|\bar{f}|_{\alpha}^{D} \\
\leqq & K|(\phi(v)-\phi(h v))-|_{2+\alpha}^{D} \\
& +|(c(x, t, v)-c(x, t, h v))-|_{\alpha}^{D}\left|\left([\phi(h v)]_{t}\right)-\right|_{\alpha}^{D}+|\bar{f}|_{\alpha}^{D} .
\end{aligned}
$$

But, $\left|\left([\phi(h v)]_{t}\right)-\right|_{\alpha}^{D} \leqq K_{1}$ for some $K_{1}>0$ and for all $h \in[0,1]$. This follows from the proof of Theorem 6.4 of $[4$, pp. 53-54]. $\phi(v)-\phi(h v)$ satisfies

$$
\begin{aligned}
L_{v}(u) & =[c(x, t, v)-c(x, t, h v)][\phi(h v)]_{t} \text { in } D+B_{T}, \\
u & =0 \text { on } \bar{B}+S .
\end{aligned}
$$

Applying Theorem 3.2 to the system (4.5), we have the estimate

$$
|(\phi(v)-\phi(h v))-|_{2+\alpha}^{D} \leqq \bar{M}|(c(x, t, v)-c(x, t, h v))-|_{\alpha}^{D}\left|\left([\phi(h v)]_{t}\right)-\right|_{\alpha}^{D} .
$$

Therefore,

$$
\begin{aligned}
|(F(v))-|_{\alpha}^{D} \leqq & K|(c(x, t, v)-c(x, t, h v))-|_{\alpha}^{D}\left|\left([\phi(h v)]_{t}\right)-\right|_{\alpha}^{D} \\
& +|(c(x, t, v)-c(x, t, h v))-|_{\alpha}^{D}\left|\left([\phi(h v)]_{t}\right)-\right|_{\alpha}^{D}+|\bar{f}|_{\alpha}^{D} \\
\leqq & (K+1) \eta|(v-h v)-|_{\alpha}^{D} K_{1}+|\bar{f}|_{\alpha}^{D} \\
\leqq & \left.\left.(K+1) \eta K_{1}|1-h|\right|_{\alpha} ^{D}\right|_{\alpha} ^{D}+|\bar{f}|_{\alpha}^{D} .
\end{aligned}
$$

We have thus proved the inequality

$$
|(F(v))-|_{x}^{D} \leqq(K+1) \eta K_{1}|1-h||\bar{v}|_{x}^{D}+|\bar{f}|_{x}^{D} .
$$


Applying Theorem 3.2 to the system (4.4), we have the estimate

$$
|(\phi(v))-|_{2+\alpha}^{D} \leqq \bar{M}|(F(v))-|_{\alpha}^{D} .
$$

Inequalities (4.6) and (4.7) yield

$$
|(\phi(v))-|_{2+\alpha}^{I)} \leqq\left[(K+1) \eta K_{1}|1-h||\bar{v}|_{\alpha}^{D}+|\bar{f}|_{\alpha}^{(D)}\right] \bar{M} .
$$

But $v \in A$ implies $|\bar{v}|_{\alpha}^{D} \leqq|\bar{v}|_{2+\alpha}^{D} \leqq 2 \bar{M}|\bar{f}|_{x}^{D}$. Choose $h$ so that $2(K+1) \eta K_{1}|1-h| \bar{M} \leqq 1$. Thus, $|(\phi(v))-|_{2+\alpha}^{D} \leqq 2 \bar{M}|\bar{f}|_{x}^{I)}$. We conclude that $\phi$ maps $A$ to $A$.

It is easy to show that $\phi$ is continuous with respect to $\left.\left.\right|^{-}\right|_{2+\alpha} ^{I)}$ in $A$. Note that if $v_{0}$ and $v$ are in $A, \phi\left(v_{0}\right)-\phi(v)$ satisfies

$$
\begin{aligned}
L_{v_{0}}(u) & =\left[c\left(x, t, v_{0}\right)-c(x, t, v)\right][\phi(v)]_{t} \text { in } D+B_{T}, \\
u & =0 \text { on } \bar{B}+S .
\end{aligned}
$$

Applying Theorem 3.2 to the system (3.8) we obtain the estimate

$$
\left|\left(\phi(v)-\phi\left(v_{0}\right)\right)-\right|_{2+\alpha}^{J J} \leqq \bar{M}\left|\left(c\left(x, t, v_{0}\right)-c(x, t, v)\right)-\right|_{\alpha}^{J}\left|\left([\phi(v)]_{t}\right)-\right|_{\alpha}^{J} .
$$

Recall that $\left|\left([\phi(v)]_{t}\right)\right|_{x}^{(I)} \leqq K_{1}$. This uniform bound, together with Lipschitz continuity of $c(x, t, u)$ with respect to $u$, and (4.9) give

$$
\left|\left(\phi(v)-\phi\left(v_{0}\right)\right)-\right|_{2: \alpha}^{I)} \leqq \bar{M} K_{1} \eta_{l}\left|\left(v_{0}-v\right)^{-}\right|_{\alpha}^{I J} .
$$

Thus, $\phi$ is continuous with respect to $\left.\left.\right|^{\cdot}\right|_{2+x}$ in $A$.

A fixed point theorem due to Schauder [2, p. 189] can be used to conclude that there exists a function $u \in A$ for which $\phi(u)=u$. We have thus proved the following theorem.

THEOREM 4.1 Assume that the conditions of Theorem 2.4 hold for the operator $L$, defined in (1.1), and suppose also that $c(x, t, u)$ is Lipschitz continuous with respect to $u$ in the $\left.\left.\right|^{\top}\right|_{x}$ norm. If $D$ has property $\left(E^{\prime}\right)$, $\psi \in \bar{C}_{2+x}(D)$, and $L \psi=f$ on $\partial B$, then there exists a solution $u$, of the nonlinear first initial-boundary value problem (4.1) and furthermore, $u \in \bar{C}_{2+\alpha}(D)$.

THEOREM 4.2. The solution $u$ obtained in Theorem 4.1 is unique.

Proof. The uniqueness of the solution to the nonlinear problem (4.1) follows from uniqueness of the solution to the linear problem of Theorem 2.4 by a well-known argument using the mean-value theorem. See for example a paper by A. P. Calderón [1, p. 35], or extend in a straightforward manner the argument given by A. Friedman [2, p. 41]. 


\section{REFERENCES}

1. A. P. Calderón, Uniqueness in the Cauchy problem for partial differential equations, Amer. J. Math. 80 (1958), 16-36. MR 21 \#3675.

2. A. Friedman, Partial differential equations of parabolic type, Prentice-Hall, Englewood Cliffs, N.J., 1964. MR 31 \#6062.

3. S. Middleman, Transport phenomena in the cardiovascular system, Wiley-Interscience Series on Biomedical Engineering, Wiley, New York, 1972.

4. M. C. Waid, Second order time degenerate parabolic equations, Trans. Amer. Math. Soc. 170 (1972), 31-56.

Department of Mathematics, University of Delaware, Newark, Delaware 19711 